\title{
Health Monitoring for Coated Steel Belts in an Elevator System
}

\author{
Huaming Lei, ${ }^{1,2}$ Guiyun Tian, ${ }^{2}$ Hui Zhao, ${ }^{1}$ Yimei Mao, ${ }^{1}$ and Zuoying Huang1 \\ ${ }^{1}$ Department of Instrument Science \& Engineering, Shanghai Jiaotong University, Shanghai 200204, China \\ ${ }^{2}$ School of Electrical, Electronic \& Computer Engineering, University of Newcastle, Newcastle NE1 7RU, UK
}

Correspondence should be addressed to Huaming Lei, hmlei@sjtu.edu.cn

Received 30 November 2011; Accepted 13 February 2012

Academic Editor: B. P. C. Rao

Copyright () 2012 Huaming Lei et al. This is an open access article distributed under the Creative Commons Attribution License, which permits unrestricted use, distribution, and reproduction in any medium, provided the original work is properly cited.

\begin{abstract}
This paper presents a method of health monitoring for coated steel belts in an elevator system by measuring the electrical resistance of the ropes embedded in the belt. A model on resistance change caused by fretting wear and stress fatigue has been established. Temperature and reciprocating cycles are also taken into consideration when determining the potential strength degradation of the belts. It is proved by experiments that the method could effectively estimate the health degradation of the most dangerous section as well as other ones along the whole belts.
\end{abstract}

\section{Introduction}

Elevator systems usually include a car and a counterweight (CW) which are suspended by tension members. A hoisting motor drives the tension members to lift the car to the desired levels within a hoistway as shown in Figure 1. Traditionally, steel ropes were used. More recently, coated steel belts have been introduced, which have a plurality of steel rope encased in a polyurethane jacket $[1,2]$. The usage of coated steel belts has several merits, such as high abrasion resistance, good flexibility and maintainability, long lifespan, low space occupation, and low noise.

With the introduction of the belts, new monitoring techniques should be developed to keep elevator systems working safely. The primary source of belts strength degradation is the cyclic bending around sheaves when the elevator is moving up and down in an elevator shaft. The tension support strength degradation is normal not uniform along the length of the belts. Those areas subject to high level or severities of bending cycles will degrade faster than areas experiencing fewer bending cycles. The two most frequent degradation forms are diameter diminution of the cords and wire broken caused by fretting wear and stress fatigue damage. This would lead to the resistance change and imply that measuring the resistance could estimate the possible degradation for the belts. Fortunately, the structure of belt ensures the possibility and convenience of measuring resistance as there is an insulating jacket and multiwire encased in the jacket. As long as some ropes were shorted at one end of the belt through a special connector, resistance could be measured at the other end.

\section{Modeling of Health Degradation}

Two basic mechanisms, fretting wear and stress fatigue damage, would cause the increasing of electrical resistance for a metallic rope. Fretting wear usually refers to the diminution in cross-sectional area of metal caused by fretting friction among the ropes. As matter of course, cross-sectional area changes of wires will naturally lead to changes in resistance. On the other hand, stress fatigue damage would cause resistance increasing based on the fact that the germination of microcracks within the material would affect the electrical conductivity $[3,4]$.

2.1. Modeling of Fretting Wear. It is quite complicated to model the fretting wear of interwire contact between wires in a rope. The wear rate is related to the contact pressure, sliding velocity, material hardness, and so on. A large number of wear models were developed in the past [5-7]. The most common wear model can be expressed by the following mathematical equation, called the Archard's law [6]:

$$
\frac{d w}{d s}=k p
$$




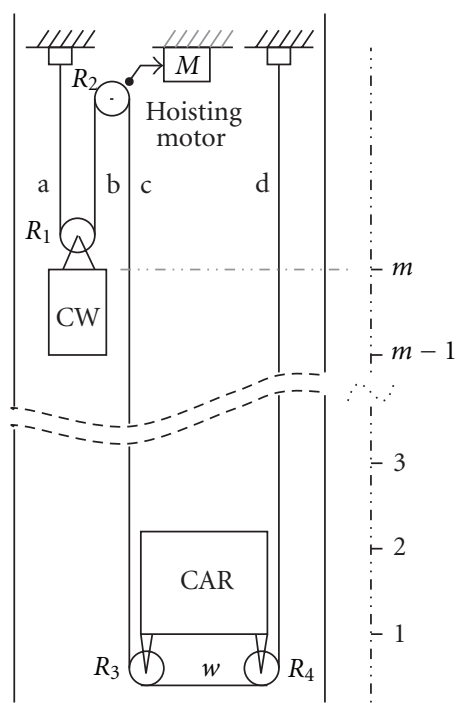

FIGURE 1: Schematic diagram of an elevator system.

Here, $w$ is the linear wear, known as, the worn volume per unit apparent contact area, $p$ is the contact pressure, $k$ is the dimensional wear coefficient, and $s$ is the sliding distance.

When $p$ is assumed to be a constant, formula (1) indicates that the wear is linearly proportional to the contact pressure $p$ in one cycle. For multicycles, the wear is linearly proportional to the number of cycles.

The belts working as tension members in an elevator system would experience two kinds of loading as axial loading and bending over sheaves. The interwire contact pressures are quite different in these two circumstances. In straight ropes the contact pressures are determined by axial loading and in bending ropes additional contact pressure would generate which depends on the diameter ratio of sheave and ropes. Based on the assumption that the maximum additional contact pressure induced due to bending of the wire rope over a sheave is proportional to axial loading, the fretting wear due to axial loading and bending could be, respectively, expressed as

$$
\begin{aligned}
& w_{0}=2 \Delta x_{0} k p_{0} n_{0}, \\
& w_{b}=2 \Delta x_{b} k p_{b} n_{b} .
\end{aligned}
$$

Here, $\Delta x$ is the stroke of cycles, and $n$ is the reciprocating cycles. The subscripts 0 and $b$ indicate the parameters are concerned with the axial loading and bending, respectively.

2.2. Modeling of Stress Fatigue Damage. When a cyclic loading is acting on metal components, fatigue microcracks would generate within the material. Fatigue damage evolution rate and cycle-creep rate are generally assumed to be functions of stress $\sigma$, damage $D$, and temperature $T$. The damage value can be approximately expressed as $[3,4,7,8]$

$$
D=1-\left[1-\frac{n}{N}\right]^{1 /(r(\beta)+1)},
$$

where $n$ is the number of reciprocating cycles and $N$ the lifespan under the specific cyclic loading. The $r(\beta)$ is a function of $\beta$ and material and temperature independent. $\beta$ is the ratio of minimum stress and maximum stress during a cycle.

If the material experiences more than one kind of load and ignores the loading sequence, the damage $D$ could be written as

$$
D=1-\left[1-\frac{\sum_{i} n_{\mathrm{e} i}}{N}\right]^{1 /(r(\beta)+1)},
$$

where,

$$
n_{\mathrm{e} i}=N\left\{1-\left(1-\frac{n_{i}}{N_{i}}\right)^{(r(\beta)+1) /\left(r\left(\beta_{i}\right)+1\right)}\right\},
$$

$n_{i}$ is the number of cycles associated with the $i$ th type of loading, and $N_{i}$ and $\beta_{i}$ are the corresponding lifespan and ratio. Formula (5) is derived based on equivalent damage principle.

2.3. Resistance Change Caused by Damage. When fretting wear or fatigue damage happens, it indicates the diameter of rope becoming smaller and micro-cracks producing within the wires and results in resistance increasing. The resistance is determined by the effective cross-sectional area of the ropes. Assuming the fretting wear and fatigue damage occurred evenly along the section which experience the same loading, the resistance before and after experiencing the loading can be written as $[3,4,8]$

$$
\begin{aligned}
& R_{0}=\frac{\rho L}{A_{0}}, \\
& R=\frac{\rho L}{A_{0}\left(1-D_{t}\right)},
\end{aligned}
$$

where $A_{0}$ is the cross-sectional area and $L$ is the length of section being researched. $A_{0}$ is a coefficient related to the cross-sectional area reduction. Obviously, the coefficient $D_{t}$ is an indication of the total damage caused by fretting wear and stress damage, it is written as

$$
D_{t}=D+\frac{w}{\Delta x A_{0}}
$$

From (6), $D_{t}$ could be expressed as

$$
D_{t}=\frac{\eta}{(1+\eta)}
$$

where $\eta$ is the resistance change rate, defined as $\eta=\Delta R / R_{0}=$ $\left(R-R_{0}\right) / R_{0}$.

From (7), the total damage could be written as

$$
D_{t}=1-\left[1-\frac{\sum_{i} n_{\mathrm{e} i}}{N}\right]^{1 /(r(\beta)+1)}+q_{0} n_{0}+q_{b} n_{b},
$$

where the $q_{0}, q_{b}$ are wear coefficients concerned with the number of reciprocating cycles. As the resistances were measured for a specified section after the section experienced different reciprocating cycles, the parameters in (9), such as $r\left(\beta_{i}\right), N_{i}, q_{0}$, and $q_{b}$, could be solved by combining the (8) and (9). 


\begin{tabular}{|c|c|c|c|c|c|c|c|c|c|c|c|c|c|c|}
\hline $\begin{array}{l}\text { Section } \\
(\mathrm{m})\end{array}$ & O & $\stackrel{n}{+}$ & 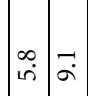 & $\exists$ & $\underset{\nearrow}{\beth}$ & 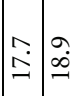 & : & הุ & \begin{tabular}{l|l}
$\infty$ & $\infty$ \\
$\stackrel{\sim}{\sim}$ & $\stackrel{\sim}{\sim}$
\end{tabular} & $\begin{array}{lll}m \\
\text { N. } \\
\end{array}$ & $\vec{\AA}$ & 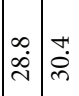 & $\ddot{m}$ & $\begin{array}{l}\text { Parameters to be } \\
\text { determined }\end{array}$ \\
\hline Sheave1 & & 16 & 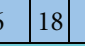 & 12 & & & & & & & & & & $w_{b 1}, N_{1}, r\left(\beta_{1}\right)$ \\
\hline Sheave2 & & & 16 & & 18 & & 1 & 12 & & & & & & $w_{b 2}, N_{2}, r\left(\beta_{2}\right)$ \\
\hline Sheave3 & & & & & & & 16 & & 18 & & 12 & & & $w_{b 3}, N_{3}, r\left(\beta_{3}\right)$ \\
\hline Sheave4 & & & & & & & & 16 & & 18 & & 12 & & $w_{b 4}, N_{4}, r\left(\beta_{4}\right)$ \\
\hline Axial loading & & & & & & & 21 & & & & & & & $w_{b 0}, N_{0}, r\left(\beta_{0}\right)$ \\
\hline $\begin{array}{l}\text { Parking } \\
\text { records }\end{array}$ & $\begin{array}{l}1 \rightarrow 2 \\
1 \rightarrow 3\end{array}$ & & $\begin{array}{l}2 \times 10 \\
6 \times 10\end{array}$ & & & $\begin{array}{l}\rightarrow 3 \\
\rightarrow 4\end{array}$ & & $\begin{array}{l}10^{4} \\
10^{4}\end{array}$ & $\begin{array}{l}3 \\
2\end{array}$ & $\begin{array}{l}\rightarrow 3 \\
2 \rightarrow 4\end{array}$ & & $\begin{array}{l}1 \times 10^{4} \\
3 \times 10^{4}\end{array}$ & & $\begin{array}{l}x \rightarrow y \text { means from } \\
x^{\text {th }} \text { floor to } y^{\text {th }} \text { floor }\end{array}$ \\
\hline
\end{tabular}

Figure 2: Sections division and the number of bending cycles $\left(10^{4}\right)$.

2.4. Degradation of a Whole Steel Belt. As mentioned before, the strength degradation is normally not uniform along the whole length of the belt. Those areas subject to high level or severities of bending cycles will degrade faster than areas experiencing fewer bending cycles. Belts working in an elevator system could be divided into several sections according to the parking operation. The electrical resistance of a whole rope within the belt after a certain operating could be written as

$$
\Delta R=\frac{R_{0}}{L} \sum_{k} \eta_{k} L_{k}=\frac{R_{0}}{L} \sum_{k} \frac{D t_{k}}{1-D t_{k}} L_{k} .
$$

Here, the subscript $k$ represents the serial number of segments.

In order to determine damage of the every section and distinguish the most dangerous segment, a couple of resistance measurements should be conducted once the belt experiences a certain amount of reciprocating cycles. Thus, the parameters in (10) could be estimated by the least squares fitting method according to the following formula:

$$
\begin{aligned}
& \min \sum_{j}\left|\Delta R_{\text {test }}-\Delta R\right|^{2} \\
& \text { s.t. } \sum_{k} L_{k}=L .
\end{aligned}
$$

Here $j$ is the serial number of measurements. Once the parameters are obtained, the damage of each section could be calculated according to formula (9).

\section{Method for Section Division}

As the elevator runs up and down in the shaft for a long period, different parts of belts would suffer different number of cycles which could be obtained from the parking records of the elevator. Some parts may experience bending only on one sheave, some may do on more than one sheave, and some would never suffer bending. Those parts experiencing the same axial loading or bending are separated as one section. The whole belt could be theoretically divided into different sections according to the structure dimension of the elevator system. Figure 2 gives an example of the sections division according to Figure 1 and the number of suffering from bending is listed. Here, the parameters are as $a=2.5 \mathrm{~m}$, $b=2 \mathrm{~m}, c=4.5 \mathrm{~m}, d=5 \mathrm{~m}, w=1.6 \mathrm{~m}, h=3.3 \mathrm{~m}$, and the total number of floor $m=4$. The variable $h$ presents height from floor to next floor.

From the results, it could be seen that different sections may suffer bending from different sheaves with different cycles. Those sections, such as [20.5-22.2], [23.8-24.3], would experience bending on more than one sheave. It could be anticipated that these sections may suffer more damage than others and become the most dangerous segments along the belt. The quantity of the damage could be estimated once obtaining the parameters by solving (11).

\section{Experiment and Results}

It would be a long-term work to conduct the experiment in a normal elevator system because the fretting wear and damage grow quite slowly even more than 30 years. In order to verify the effectiveness of the method, accelerated fatigue experimental work was conducted on a belt of length $5.541 \mathrm{~m}$ and with 6 core ropes whose diameter is $2.3 \mathrm{~mm}$. The loaded belt experienced repeated bending on two sheaves with the diameter $110 \mathrm{~mm}$ and approximate contact angle 90 degrees. Figure 3 shows the schematic diagram of mechanical structure for fatigue experiment.

Temperature was recorded as well as the resistance in the experiment. It is found that the TCR (temperature coefficient of resistance) of the belt would not change after fatigue experiment. This is an important result for estimating damage under different temperature. The temperature and TCR are used to rectify the electrical resistance and get an identical evaluation criterion under an assumed temperature.

The resistances for one of the ropes that experienced different cycles were measured by a high-accuracy multimeter at temperature $26^{\circ} \mathrm{C}$. The parameters were estimated by (11) and the estimated resistance curve is shown in Figure 4 compared with the measured data. By using the parameters, the damage for each section could be calculated. As a contrast, the resistances of each section were measured after 100,000 cycles. The calculated resistance is in good agreement with the actual measured ones as shown in Table 1. The measured $\Delta R / R_{0}$ rate is in good agreement with the inferred damage $D_{t}$. 
TABLE 1: Data of fatigued belts according to different sections.

\begin{tabular}{|c|c|c|c|c|c|c|}
\hline Segment no. & $\mathrm{I}$ & II & III & IV & $\mathrm{V}$ & Tot. or aver. \\
\hline Length $L(\mathrm{~m})$ & 2.279 & 0.478 & 0.461 & 0.486 & 1.837 & 5.541 \\
\hline Resistance $R(\mathrm{~m} \Omega)$ & 217.3 & 46.5 & 48.3 & 47.8 & 175.2 & 535.1 \\
\hline Pre-fatigued $R_{0 i}(\mathrm{~m} \Omega)$ & 216.1 & 45.3 & 43.7 & 46.1 & 174.2 & 525.4 \\
\hline Measured $\Delta R(\mathrm{~m} \Omega)$ & 1.2 & 1.2 & 4.6 & 1.7 & 1.0 & 9.7 \\
\hline Normalized $R(\mathrm{~m} \Omega / \mathrm{m})$ & 95.35 & 97.26 & 104.81 & 98.32 & 95.37 & 96.57 \\
\hline Measured $\Delta R / R_{0}(\%)$ & 0.56 & 2.65 & 10.53 & 3.69 & 0.57 & 1.85 \\
\hline Inferred $\Delta R(\mathrm{~m} \Omega)$ & - & 1.41 & 4.59 & 1.44 & - & 7.44 \\
\hline Inferred Damage $D_{t}(\%)$ & - & 3.03 & 9.51 & 3.03 & - & - \\
\hline Condition & \multicolumn{6}{|c|}{$R_{0}=94.83 \mathrm{~m} \Omega / \mathrm{m}$ at temperature $T_{0}\left(26^{\circ} \mathrm{C}\right)$} \\
\hline
\end{tabular}

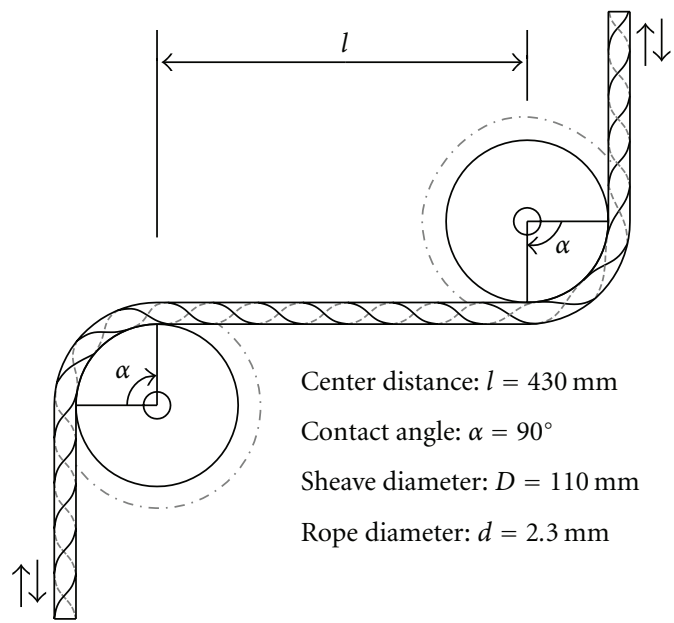

FIGURE 3: Mechanical structure diagram for fatigue experiment.

On the condition that the belt is working under normal condition and will not suffer occasional injure, such as sharp twisting or hammer hitting, the presented method is effective and reliable for the damage evaluation and life prediction. Furthermore, from the measured $\Delta R / R_{0}$, it can be inferred that the fretting wear and stress damage that acted on section II and section IV of the two sheaves are not exactly the same. This may be attributed to the inconsistent installation of the two sheaves.

\section{Conclusions}

An inspection and health monitoring method for steel wire belts is presented and proved by fatigue experiment. It is an effective method to monitor the stress degradation for belts by measuring the electrical resistance of the embedded rope. It should be noted that the established model is based on a lot of assumptions and ignores the loading sequence. The fatigue experiment is built on an accelerated aging setup, which leads to that most of the change in resistance may be caused by stress fatigue. Although, in an elevator system, the belts experience high-cycles fatigue, resistance increase caused by fretting wear may take the dominant role. All these should be considered more carefully in an actual application for elevators. In the future, more efforts would be put on

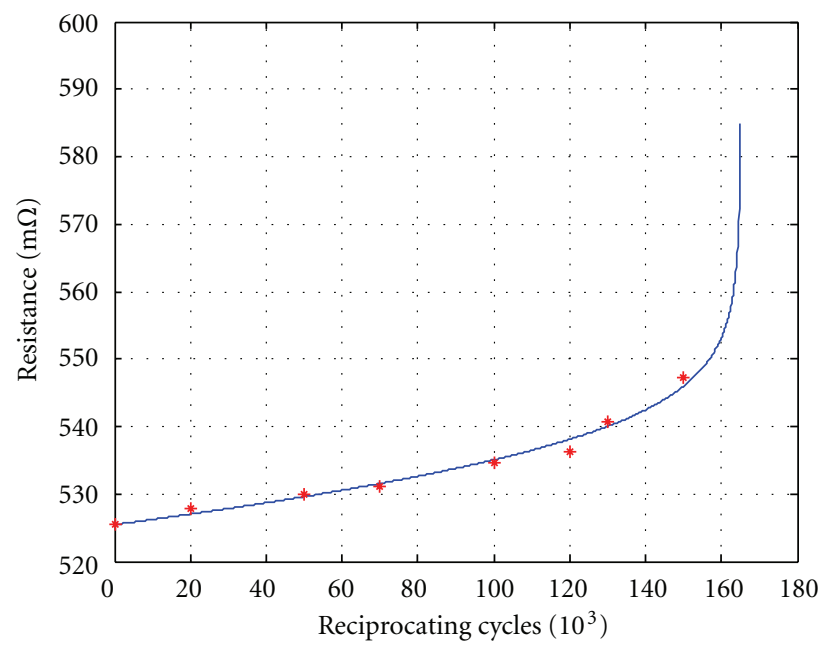

Figure 4: The estimated curve of the damage and the change of resistance for the belts.

using the magnetic flux leakage or eddy current methods to test the belts, and results will be compared with the inferred damage, to make the presented model more applicable.

\section{Acknowledgments}

The author would like to acknowledge Shanghai Mitsubishi Elevator Company for the experiment supports and the China Scholarship Council funding for the academic visiting to Newcastle University. Thanks also should go to the National Natural Science Foundation of China (NSFC-60702045) for the financial support.

\section{References}

[1] P. Wesson John and R. Gurvich Mark, "Method of making an elevator belt," WIPO Patent Application, WO/2010/056247 A1. 2010.

[2] Y. Konishi, A. Ueno, A. Wake, and K. Yuasa, "Elevator drive belt [p]," WO-02/064482. 2004.10.

[3] W. June, "A continuum damage mechanics model for low-cycle fatigue failure of metals," Engineering Fracture Mechanics, vol. 41, no. 3, pp. 437-441, 1992. 
[4] J. Jusheng and X. Jinquan, "The research of damage in fatigue by means of resistance of metal materials," Journal of Mechanical Strength, vol. 21, pp. 232-234, 1999.

[5] I. I. Argatov, X. Gómez, W. Tato, and M. A. Urchegui, "Wear evolution in a stranded rope under cyclic bending: implications to fatigue life estimation," Wear, vol. 271, no. 11-12, pp. 28572867, 2011.

[6] A. Cruzado, M. Hartelt, R. Wäsche, M. A. Urchegui, and X. Gómez, "Fretting wear of thin steel wires. Part 1: influence of contact pressure," Wear, vol. 268, no. 11-12, pp. 1409-1416, 2010 .

[7] D. K. Zhang, S. R. Ge, and Y. H. Qiang, "Research on the fatigue and fracture behavior due to the fretting wear of steel wire in hoisting rope," Wear, vol. 255, no. 7-12, pp. 1233-1237, 2003.

[8] P. Starke, F. Walther, and D. Eifler, "PHYBAL_a new method for lifetime prediction based on strain, temperature and electrical measurements," International Journal of Fatigue, vol. 28, no. 9, pp. 1028-1036, 2006. 

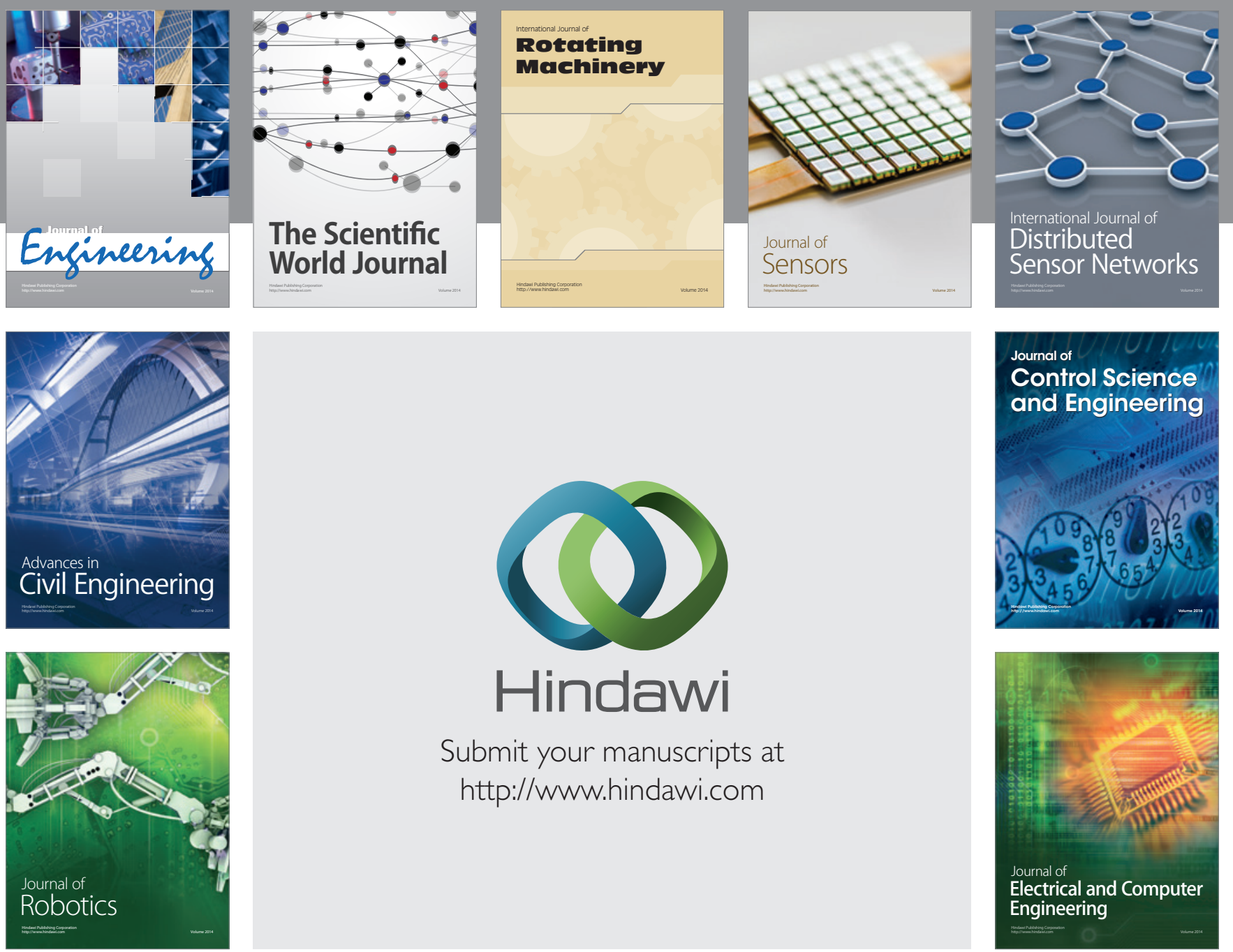

Submit your manuscripts at

http://www.hindawi.com
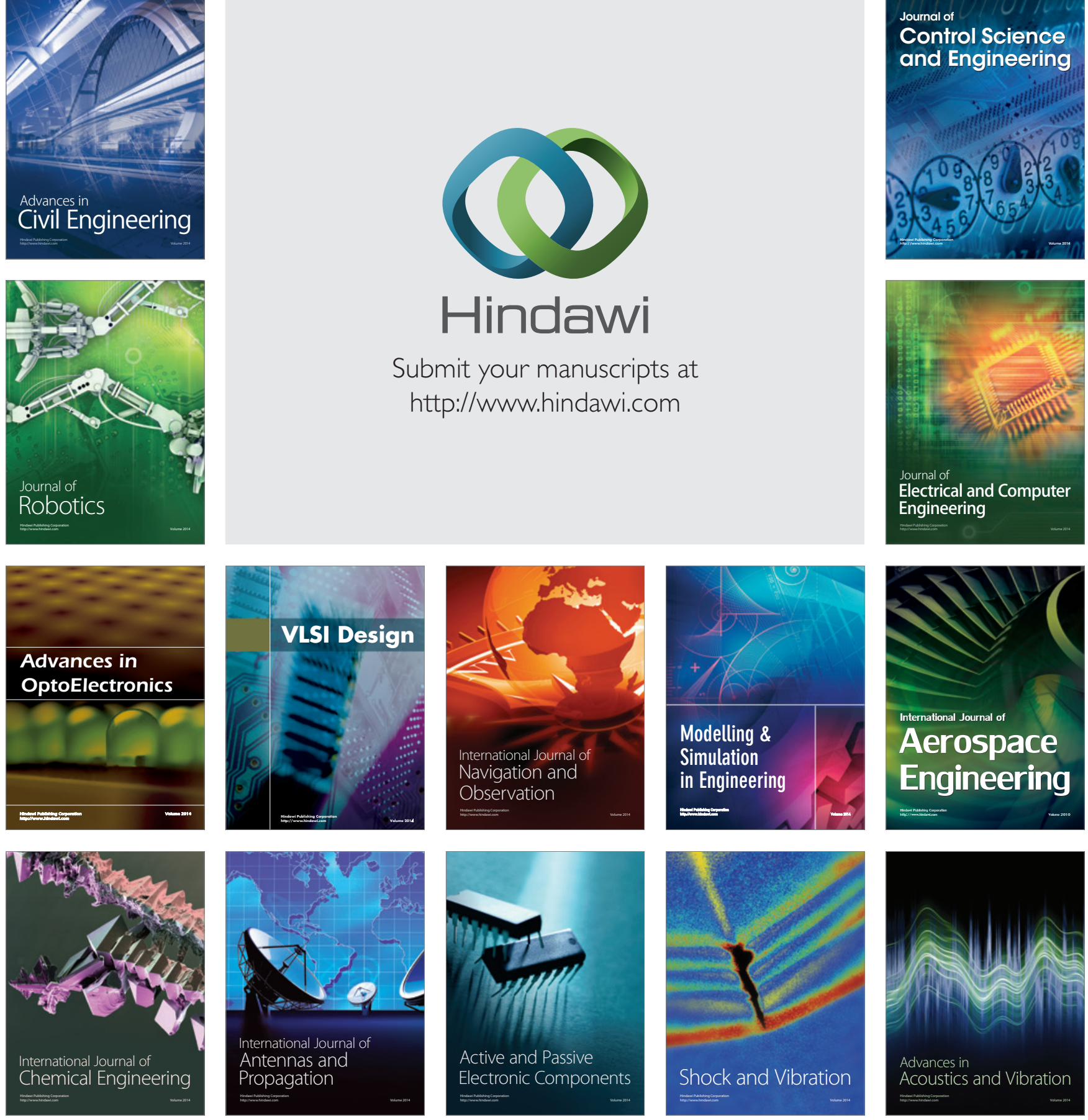